\title{
MHD equilibria and triggers for prominence eruption
}

\author{
Yuhong Fan
}

\begin{abstract}
Magneto-hydrodynamic (MHD) simulations of the emergence of twisted magnetic flux tubes from the solar interior into the corona are discussed to illustrate how twisted and sheared coronal magnetic structures (with free magnetic energy), capable of driving filament eruptions, can form in the corona in emerging active regions. Several basic mechanisms that can disrupt the quasi-equilibrium coronal structures and trigger the release of the stored free magnetic energy are discussed. These include both ideal processes such as the onset of the helical kink instability and the torus instability of a twisted coronal flux rope structure and the non-ideal process of the onset of fast magnetic reconnections in current sheets. Representative MHD simulations of the non-linear evolution involving these mechanisms are presented.
\end{abstract}

\section{Introduction}

Prominences/filaments are major precursors or source regions of coronal mass ejections (CMEs) as indicated by the observed close association between prominence/filament eruptions and CMEs [70, 86, 45]. It is suggested that most CMEs are the result of the destabilization and eruption of a prominence and its overlying coronal structure, or of a magnetic structure capable of supporting a prominence [86]. On the large scale, prominences/filaments (either in strong active region nests or in the weak fields of decaying active regions) represent stable structures that can exist over long periods of time (days), that are much longer than the Alfvén crossing time (minutes), before they suddenly erupt. Thus, on the large scale for the prominence structure as a whole, prominence magnetic fields represent magneto-static equilibria in the corona. Although on smaller scales, prominence plasma exhibits continuous,

Yuhong Fan

National Center for Atmospheric Research, 3080 Center Green Dr., Boulder, CO 80301, yfanducar. edu. 
on-going dynamic and turbulent behavior within the structure (e.g. [16, 24, 57] and see also [49]).

Given the low plasma $\beta$ in the lower solar corona, where $\beta$ denotes the ratio of the plasma pressure over the magnetic pressure, and if the gravitational force of the prominence mass is not significant to distort the magnetic fields on which it resides, one class of models considers prominence magnetic fields as approximately force free equilibrium structures with field aligned electric current, i.e.

$$
\nabla \times \mathbf{B}=\alpha \mathbf{B}
$$

where $\alpha$ is the torsion or twist parameter being constant along each field line but generally different for different field lines. The field aligned current, which manifests as the twist or shear of the magnetic field represents the free magnetic energy (in excess of of the potential field energy) stored in the equilibrium fields that can be released to drive the eruption.

Another class of models consider the weight of the prominence mass as playing a significant role in both the energy storage and release of the prominence magnetic structures (e.g. [51, 60, 61, 38, 59, 73, 89, 90]). In these models a significant local dip or distortion in the field lines can be created due to the weight of the prominence plasma, which produces significant cross-field current and hence additional free magnetic energy. A sudden removal of the prominence mass through some physical mechanisms can therefore release the stored free magnetic energy and drive eruptions.

Due to the nearly frozen-in evolution of the large scale coronal magnetic field in the highly conducting plasma of the solar atmosphere and corona, the magnetic helicity, a physical quantity that measures the topological complexity of the magnetic field (such as the linkage and/or twistness of the field) is nearly conserved (e.g. [15, 14]). In a volume $V$ with all magnetic flux closed within the volume, the magnetic helicity is given by $H=\int_{V} \mathbf{A} \times \mathbf{B} d V$, where $\mathbf{A}$ is the vector potential of the magnetic field $\mathbf{B}$ in $V$, i.e. $\mathbf{B}=\nabla \times \mathbf{A}$, and it can be shown that $H$ is invariant to any gauge transformation of $\mathbf{A} \rightarrow \mathbf{A}+\nabla \chi$ with $\chi$ being an arbitrary scalar function of position, and thus $H$ is a well defined quantity. For example, two linked, untwisted closed flux tubes with fluxes $\Phi_{1}$ and $\Phi_{2}$ respectively, have a magnetic helicity of $H=2 \Phi_{1} \Phi_{2}$, and a uniformly twisted closed magnetic torus with $T$ winds of field line rotation about the axis over the length of the torus and with a total toroidal flux of $\Phi$ has a magnetic helicity $H=T \Phi^{2}$ ([15]). For the solar corona, we do not generally have an isolated, closed magnetic flux system and the magnetic flux is generally threading through the photosphere. Therefore a relative magnetic helicity for the magnetic field above the photospheric $z=0$ is defined ([15])

$$
H_{r}=\int_{z>0}\left(\mathbf{A}+\mathbf{A}_{p}\right) \cdot(\mathbf{B}-\mathbf{P}) d V,
$$

where $\mathbf{B}$ is the magnetic field in the unbounded half space above $z=0, \mathbf{A}$ is the vector potential for $\mathbf{B}, \mathbf{P}$ is the reference potential field having the same normal flux distribution as $\mathbf{B}$ on the $z=0$ boundary, and $\mathbf{A}_{p}$ is the vector potential for $\mathbf{P}$. 
The relative magnetic helicity $H_{r}$ is invariant with respect to the gauges for $\mathbf{A}$ and $\mathbf{A}_{p}$, and is thus a well-defined measure of the linkage or twistness of the coronal magnetic field ([15, 25]). The evolution of $H_{r}$ in the corona is given by (e.g. [25]):

$$
\frac{d H_{r}}{d t}=-2 \int_{S} \mathbf{A}_{p} \times(\mathbf{v} \times \mathbf{B}) \cdot \hat{\mathbf{z}} d S+\left(\frac{d H_{r}}{d t}\right)_{\text {diss. }},
$$

where the first term on the right-hand-side corresponds to integration of helicity flux over the photospheric surface and the 2nd term corresponds to dissipation of $H_{r}$ in the corona. In the above $\mathbf{A}_{p}$ is the uniquely determined vector potential of the potential magnetic field with the gauge conditions: $\mathbf{A}_{p} \cdot \hat{\mathbf{z}}=0$ on $S$, and $\nabla \cdot \mathbf{A}_{p}=0$ in the corona above $S$. It is shown that the helicity dissipation (2nd term) is negligible for the nearly frozen-in evolution of the large scale corona even including magnetic reconnections during flares ([14]). Such constraint of magnetic helicity conservation is playing an important role in the energy storage and ultimate eruption of the filament/prominence magnetic fields as described by [90]. The net helicity transported into the corona through the photosphere (first term in the right-hand-side of eq. (3) via flux emergence from the interior cannot be flared away and therefore the free magnetic energy cannot be completely dissipated down to the minimum energy potential field level ([87, 90, 25]). The observed hemispheric pattern of the chirality of filament channels (see section 3.1 in [29]) is directly related to the sign of magnetic helicity contained in the filament channel magnetic fields, where a dextral (sinistral) filament channel preferred in the northern (southern) hemisphere contains dominantly negative (positive) helicity or left-handed (right-handed) twist. The hemispheric pattern of the helicity of filament channels has its origin in the accumulation of the helicity in emerging active regions (e.g. [90, 64, 88] and see also [63]), which are observed to also show preferentially negative (positive) twist in the northern (southern) hemisphere, and this sign preference does not change with the solar cycles. Such accumulation of net helicity in each hemisphere is ultimately removed by the bodily ejection of the filament/prominence magnetic fields as coronal mass ejections ([90]).

The main questions to be addressed in this chapter are (1) how sheared or twisted structures form in the corona as a result of magnetic flux emergence? and (2) what are the mechanisms that lead to the sudden disruption of the quasi-equilibrium coronal structures and an explosive release of the free magnetic energy? Question (1) is discussed in section 2 with focus on understanding the formation of strongly twisted emerging active regions that develop X-ray sigmoids and/or sigmoid shaped filaments. Example MHD simulations of the emergence of a twisted flux tube from the solar interior into the atmosphere and corona are shown to demonstrate how helicity and free magnetic energy are transported into the corona. In section 3 the basic mechanisms that can trigger the dynamic eruption of the twisted/sheared coronal magnetic structures are discussed and several MHD simulations of the non-linear evolution involving these mechanisms are presented. It is argued that current sheet formation and magnetic reconnection are playing an important role in all stages of 
the evolution of the prominence/filament magnetic fields, before, during and after the eruption.

\section{Emergence of twisted magnetic fields and build up of free energy and helicity in the corona}

Observations suggest that flare productive active regions are associated with the emergence of twisted magnetic flux from the solar interior (see review by [77]). Vector magnetic field observations of the photospheric layer of such active regions show that the transverse magnetic field at the polarity inversion lines (PILs) tend to be strongly sheared, i.e. tends to be closer to being parallel to the PILs rather than being perpendicular as expected for a potential field configuration (e.g. review by [77]). And sometimes the transverse magnetic field shows an "inverse-polarity configuration" pointing from the negative polarity to the positive polarity, indicating a concave upturning field configuration at the PILs (e.g. [55, 20, 71]). Continuous observations by SOT of Hinode studied by [71] have found a temporal evolution of the transverse magnetic field at the PIL from a "normal-polarity" configuration (pointing from the positive to negative polarity as expected for a convex arcade loop field) to an "inverse-polarity configuration", which was interpreted as the signature of the emergence of a helical flux rope through the photosphere. Flare productive active regions also often show velocity shear at the PILs and rotating sunspots, indicative of transport of twist into the solar corona (e.g. [19, 91, 48]), and develop sigmoidshaped X-ray loops and sigmoid-shaped filaments in the corona (e.g. [22, 44], see also Figure 9 in [29]).

MHD simulations have shown that the emergence of a twisted magnetic flux tube from the interior into the solar atmosphere and corona can qualitatively explain many of these commonly observed features associated with strongly flaring active regions (e.g. [66, 67, 9, 30, 35, 37, 36, 53]). Figure 1 shows the results from one example simulation ([30]) of the 3D coronal magnetic field structure and the photospheric flux emergence patterns produced by a subsurface twisted flux tube whose central segment rises buoyantly to the photosphere and emerges into the atmosphere and the corona as a result of the non-linear evolution of the magnetic buoyancy instability. It is found that the twisted subsurface flux tube does not emerge as a whole into the corona. While the upper parts of the helical field lines of the subsurface tube expand into the atmosphere due to the onset of the magnetic buoyancy instability, the bottom U-shaped portions of the winding field lines remain anchored at and below the photosphere layer by the weight of the plasma. Nevertheless, the simulations (e.g. [66, 67, 9, 30, 53]) show that a flux rope structure with field lines winding about each other and with sigmoid-shaped, dipped, core field lines eventually forms in the corona (top-left panel in Figure 11). It is found that the Lorentz force drives both shear flows at the PIL ([67]) and rotational motions in each of the polarity concentrations (reminiscent of rotating sunspots) as shown in the bottom right panel of Figure 1 Such vortical motions are caused by a gradient of the torsion or twist 


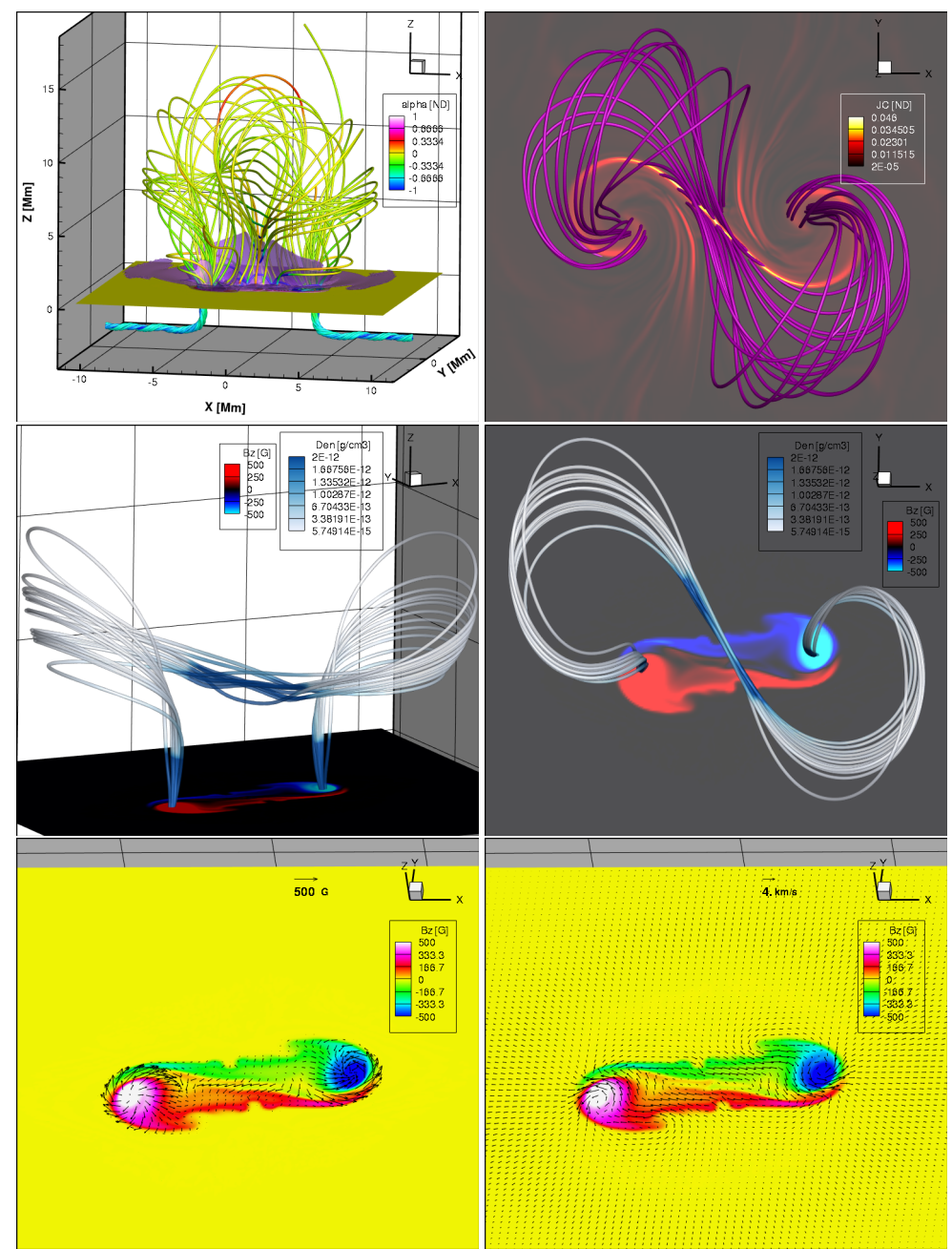

Fig. 1 Results from an example MHD simulation of the dynamic emergence of a twisted subsurface flux tube into the solar atmosphere and corona as described in [30]. Top-left panel shows the 3D coronal magnetic field structure with field lines colored by the torsion parameter $\alpha$. The purple surface is the iso-surface of electric current density $J$, outlining regions of high current concentration. Top right panel shows sample field lines (in purple) going through the central current sheet. The background color image shows a horizontal cross-section of the current density $J$ (the color map is for $J$ ) at $3 \mathrm{Mm}$ above the photosphere. The two middle panels show two perspective views of a set of field lines with central dips in the corona at a height about $5 \mathrm{Mm}$ above the photosphere. The field lines are colored by the density, showing enhanced density at the dips. The two bottom panels show the vertical magnetic field pattern (color image) with arrows of the transverse magnetic field (bottom left panel) and horizontal velocity field (bottom right panel).

$\alpha \equiv((\nabla \times \mathbf{B}) \cdot \mathbf{B}) / \mathbf{B}^{2}$ along the field lines from the interior to the corona (see the $\alpha$ coloring of the field lines in the top left panel of Figure 1) due to the great expan- 
sion and stretching of the emerged coronal fields $([58,30])$. The shear and vortical motions are the major means by which twist or magnetic helicity are continually transported from the interior flux rope into the corona in the emerging region. [30] found that with the continued twisting of the emerged field lines by the vortical motions at their footpoints, the field lines above the PILs rotate and change their orientation from an initial "normal-polarity" configuration into an "inverse-polarity" configuration (see bottom left panel of Figure 1), leading to the formation of the sigmoid-shaped dipped core fields (see the example field lines shown in the middle panels of Figure 11). This would explain the observed rotation of the transverse field at the PIL described in [71]. With continued transport of twist into the corona, the sigmoid-shaped core field also begins to rise upward into the corona, causing an underlying sigmoid-shaped vertical current sheet to form (as outlined by the purple iso-surface of the current density $J$ in the top left panel of Figure 11). Reconnections in this vertical current sheet are of the "tether-cutting" type (e.g. [69]) that disengage the anchoring of the field lines and allow the coronal flux rope structure to rise further in the corona (e.g. [67, 30], in some cases leading to eruptive behavior (e.g. [8, 10]). The top right panel of Figure 1 shows the horizontal cross-section of $J$ at 3 $\mathrm{Mm}$ above the photosphere showing the sigmoid-shaped current concentration, and the sampled field lines (in purple) going through the strong current concentration may correspond to the observed sigmoid-shaped X-ray loops.

More recent simulations ([53]) of twisted flux tube emergence incorporating a pre-existing dipole coronal field found clearly the formation of a stably confined coronal flux rope structure with sigmoid shaped dipped core fields and an underlying current sheet similar to that found in Figure 1 . They confirm the results that shear and sunspot rotation are driven by twisted flux tube emergence and they can cause the formation of stable sigmoids prior to a solar flare. Simulations that incorporate magneto-convection ([36]) in the interior layer also found that shear flows at the PILs and sunspot rotation driven by the Lorentz force are the major means twist and free-magnetic energy are transported from the interior into the corona (Figure 22. It is found that sigmoid-shaped sheared fields are built up in the corona, but the formation of a coherent flux rope structure with dipped fields and inverse-polarity configuration has not been seen in these simulations.

\section{Initiation mechanisms for eruption:}

Due to the fast Alfvén speed in the lower solar corona $\left(\sim 1000 \mathrm{~km} \mathrm{~s}^{-1}\right)$, the process of magnetic flux emergence characterized by a photospheric flow speed of order a few $\mathrm{km} / \mathrm{s}$ represents a slow driving or change of the coronal magnetic field. Thus the resulting twisted coronal structure (as discussed in the previous section) that forms is expected to evolve quasi-statically through a sequence of near force free equilibria as it is being driven slowly at the foot points by the continued shearing and twisting produced by the flux emergence. The reason that the quasi-equilibrium coronal structures suddenly erupt as flares and/or coronal mass ejections is still under de- 

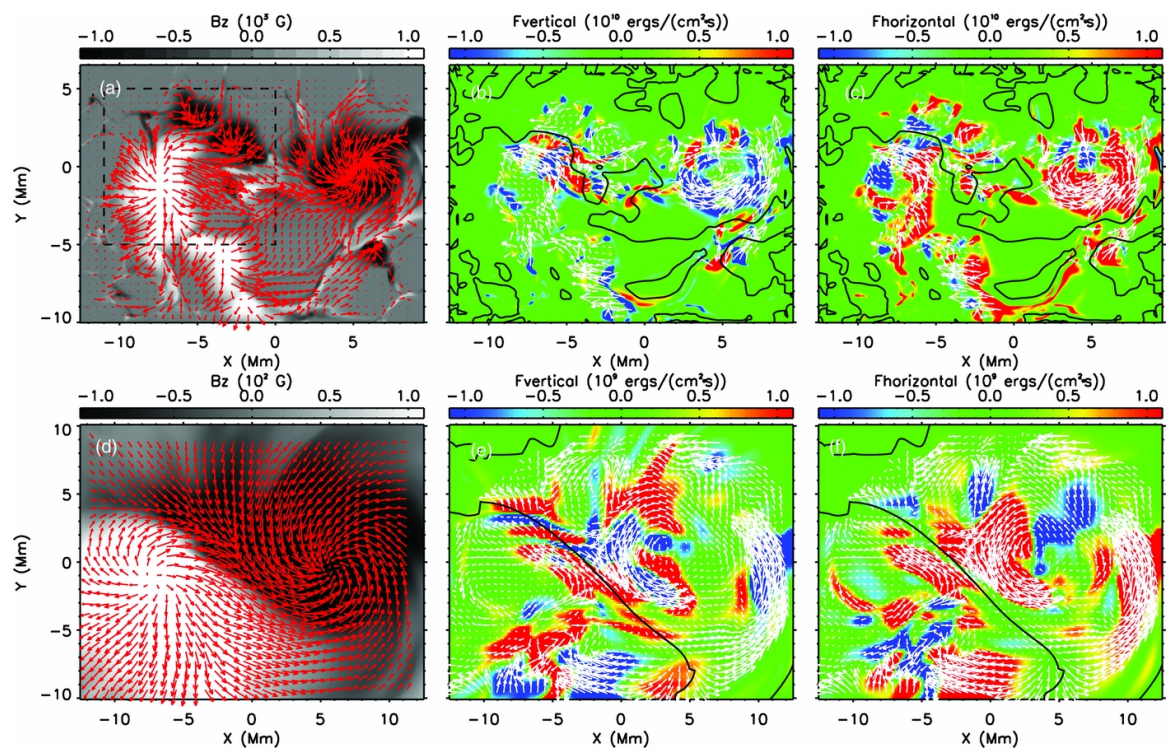

Fig. 2 Flux emergence pattern from a simulation of the emergence of a twisted flux tube from the interior into the atmosphere and corona including magneto-convection in the interior layer by [36]: Grayscale image of $B_{z}$ and red arrows of the transverse magnetic field (a), Poynting flux $F_{\text {vertical }}$ due to direct vertical motions with white arrows of horizontal velocity fields (b), and Poynting flux $F_{\text {horizontal }}$ due to horizontal flow fields with white arrows of horizontal velocity (c), on the photosphere. (d),(e), and (f) are the same as (a), (b), and (c) respectively but at a height of $z=3$ $\mathrm{Mm}$ in the corona. PIL is shown by the black line. One clearly sees shear flows at the PIL, and a prominent rotation of the negative polarity spot. Transport of magnetic energy into the corona is clearly dominated by the component due to the horizontal motions (shear and sunspot rotation). Figure from [36] reproduced by permission of the AAS.

bate. The mechanism that leads to the loss of a stable equilibrium and triggers the energy release and eruption may be purely ideal or involve non-ideal processes such as magnetic reconnections (e.g. [40]). One likely possibility is the onset of an idealMHD instability or a sudden loss of an ideal-MHD equilibrium. Such mechanisms can naturally account for the fast Alfvén time scales for the onset of the eruptions.

\subsection{Ideal MHD instabilities and loss of equilibrium of force free coronal flux ropes}

A coronal magnetic field may suddenly erupt if the (force free) equilibrium becomes unstable to perturbations, i.e. if the resulting forces produced by the perturbations make the perturbations grow rather than restoring the equilibrium, or if there is no more neighboring equilibria in the evolution of the force free coronal magnetic field. Both types of theoretical analysis of (1) the ideal linear instabilities of a force 
free equilibrium (e.g. [46], [52], [47], [26]) or (2) the catastrophic loss of neighboring force free equilibrium solutions beyond a certain value of some evolutionary parameter (e.g. [41], [54], [26]) have been carried out.

Two current-driven instabilities that have been extensively studied as likely triggers for flares and eruptions are the helical kink instability and the so called "torus instability" associated with a twisted flux rope. A force free cylindrically symmetric twisted flux tube of infinite length is shown to be always unstable to the helical kink instability ([7]). The kink instability can be suppressed if the ends of the cylindrical flux tube are line-tied such that within the finite length of the flux tube the total twist is not too high ([75]). Thus anchoring of the footpoints of the coronal loops by the heavy plasma of the photosphere is stabilizing for the coronal magnetic field. Again considering cylindrically symmetric force free magnetic flux tubes line-tied at both ends, [46] show that for a uniformly twisted flux tube the kink instability sets in if the angle each field line is twisted in going from one end to the other exceeds 2.49 $\pi$, or about 1.25 full rotations.

On the other hand, the torus instability is an expansion instability associated with a toroidal current ring held in equilibrium in an external potential magnetic field ([52], [26]). The stability of such equilibrium configurations has been studied in Tokamaks (e.g. [13]). The torus instability for an arched coronal flux rope confined by an external potential field has been demonstrated using an analytic model ([80], [47]) of an approximately force free coronal magnetic field as shown in Figure 3 . hereafter referred to as the T\&D (Titov and Demoulin) flux rope model. The force

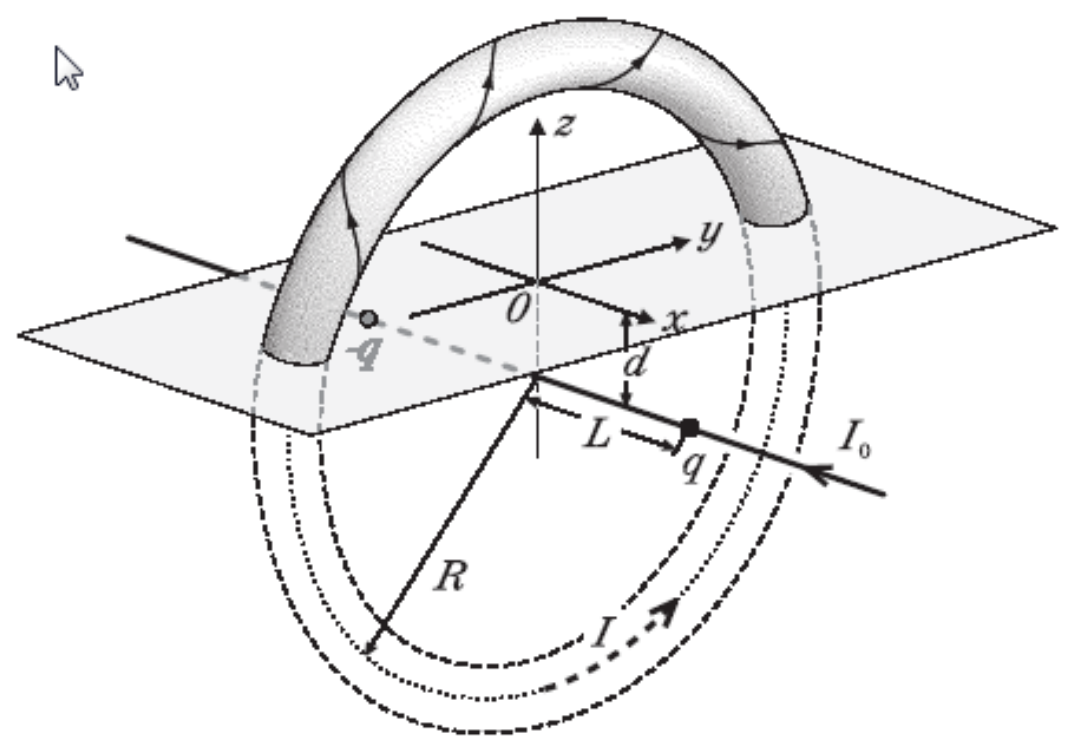

Fig. 3 The T\&D (Titov and Demoulin) force free coronal flux rope model from [80]. Figure reproduced with permission by Astron. \& Astrophys. 
free coronal magnetic field above the photosphere is constructed using three sources: a circular flux rope with a thin total current $I$, a pair of charges $q$ and $-q$ below the photosphere, and a line current $I_{0}$ below the surface. The subsurface sources are just virtual sources for the analytic construction of the normal magnetic flux at the photosphere, and are not reality. With such construction, the forces at the apex of the flux rope ([80]) can be decomposed into an outward hoop force, corresponding to the self-repulsive force of the circular current $I$, and an inward confining force acting on the flux rope current due to the potential field $B_{p}$ produced by the charges. Note, the subsurface line current $I_{0}$ is introduced as a means of controlling the pitch of the magnetic field in the vicinity of the coronal current and it does not exert a force on the coronal current $I$ because the field $I_{0}$ generates is parallel to $I$. In equilibrium the outward hoop force and the inward confining force due to the potential magnetic field should balance. [80] considered a sequence of equilibrium states with increasing major radius $R$, which could be viewed as a quasi-static emergence of the flux rope. They found that the equilibrium becomes unstable to an expansion $\delta R$, when $R$ reaches a critical size with respect to the separation of the charges, where the decline of the potential field $B_{p}$ with $R$ becomes sufficiently steep, as measured by a decay index of $n \equiv-d \ln B_{p} / d \ln R>1.5$, such that the decline of the confining force by the potential magnetic field becomes faster than the decline of the outward hoop force. [80]'s calculation of the instability considered an azimuthally symmetric expansion $\delta R$, and therefore does not enforce line-tying by the heavy photosphere during the time scale for the onset of the instability. Later improved calculation of the torus instability for the T\&D flux rope equilibrium by [47] considered perturbations that truly enforce anchoring of the coronal flux rope on the photosphere by using a subsurface image current to wipe out the change on the photosphere magnetic field produced by the change of the coronal current. They found similar results, that there is a critical height for the apex of the coronal flux rope, above which stable equilibrium of the flux rope confinement is not possible.

[26] examined both the loss of equilibrium and the torus instability of coronal flux ropes with concentrated thin current channels in the corona of either a straight or circular shape. They found that a global instability of the magnetic configuration is present when the current channel is located at a coronal height, $h$, large enough so that the decay index of the potential field, $n \equiv-d \ln B_{p} / d \ln h$, is larger than a critical value that is in the range of 1 to 1.5. They found that when a loss of equilibrium occurs the magnetic configuration is also ideally unstable to the torus instability.

\subsection{MHD simulations of the eruption of coronal flux ropes}

The above analytical studies of the ideal linear instabilities and loss of equilibrium of coronal force free magnetic fields are necessarily limited to highly idealized field configurations with a high degree of symmetry. To study realistic three-dimensional coronal magnetic field configurations and the non-linear evolution of the instabilities 
and loss of equilibrium, MHD simulations are important tools to provide physical insight.

[84, 82] have performed detailed studies of the helical kink instability of an arched coronal flux rope, line-tied to the photosphere, using the T\&D analytical flux rope model as the initial state in three-dimensional ideal MHD simulations. They have shown that this model relaxes to a numerical equilibrium very close to the initial analytical model in the case of subcritical twist and that the helical kink instability develops for supercritical twist in the anchored flux rope. It is found that the non-linear development of the kink instability with supercritically twisted initial flux ropes can lead to either confined (failed) eruptions or CME like ejective eruptions depending on how rapidly the overlying field above the flux rope decreases with height. By using a certain parameter setup of the T\&D flux rope with a supercritical initial twist, [82] is able to reproduce both the development of the helical shape and the rise profile of a confined (or failed) filament eruption observed on 27 May 2002 (see Figure 4). The eruption of the kinked flux rope is halted by the strong overlying field and reconnection outflows in the current sheet above the flux rope causes expansion of the top part of the flux rope in the lateral directions as seen in both the simulation and the observation. A strong vertical current sheet also develops under the kinked flux rope between the two legs of the kinked loop (e.g. [33, 82]), which is consistent with the coronal hard X-ray emission near the crossing of the loop legs observed during the flare ([56]). The similarities between the helical shape of erupting filament/prominence in many observed events and the magnetic field morphology produced by MHD simulations of the evolution of kink unstable coronal flux ropes (e.g. [82, 33, 43]) suggest that the onset of the kink instability is a viable initiation mechanism for triggering many of such events, and that flux ropes with substantial twist can exist or form in the solar corona prior to eruption.

Further using initial T\&D flux rope configurations in the parameter regime where the flux rope is subcritical for the onset of the helical kink instability but supercritical for the expansion torus instability, [83] studied the non-linear evolution of the torus instability of line-tied coronal flux rope embedded in a potential field with varying decline profiles with height. It is found that the critical decay index $n_{\mathrm{cr}}$ of the potential field for the torus instability of the line-tied 3D flux rope is similar to the analytical result for a freely expanding toroidal current ring ([52]), with $n_{\mathrm{cr}} \sim 1.5$. It is also found that the acceleration profile for the eruption of the unstable flux rope depends on the steepness of the field decrease, corresponding to fast CMEs for rapid decrease (as typical of compact active regions) and to slow CMEs for gradual decrease (as typical of quiescent filament eruptions).

The above MHD simulations of the helical kink instability and torus instability of coronal flux ropes have all started out with initial configurations (T\&D analytical models) that are already supercritical for the onset of the instabilities. For studying how unstable configurations come to being and the transition from the quasi-static buildup phase to the dynamic eruptive phase, there have been many MHD simulations of the buildup and eruption of coronal flux ropes driven at the lower boundary by various flux transport processes including: a slow imposed flux emergence (e.g. [3, 34, 31, 32, 23], shearing and twisting motions in conjunction with flux 

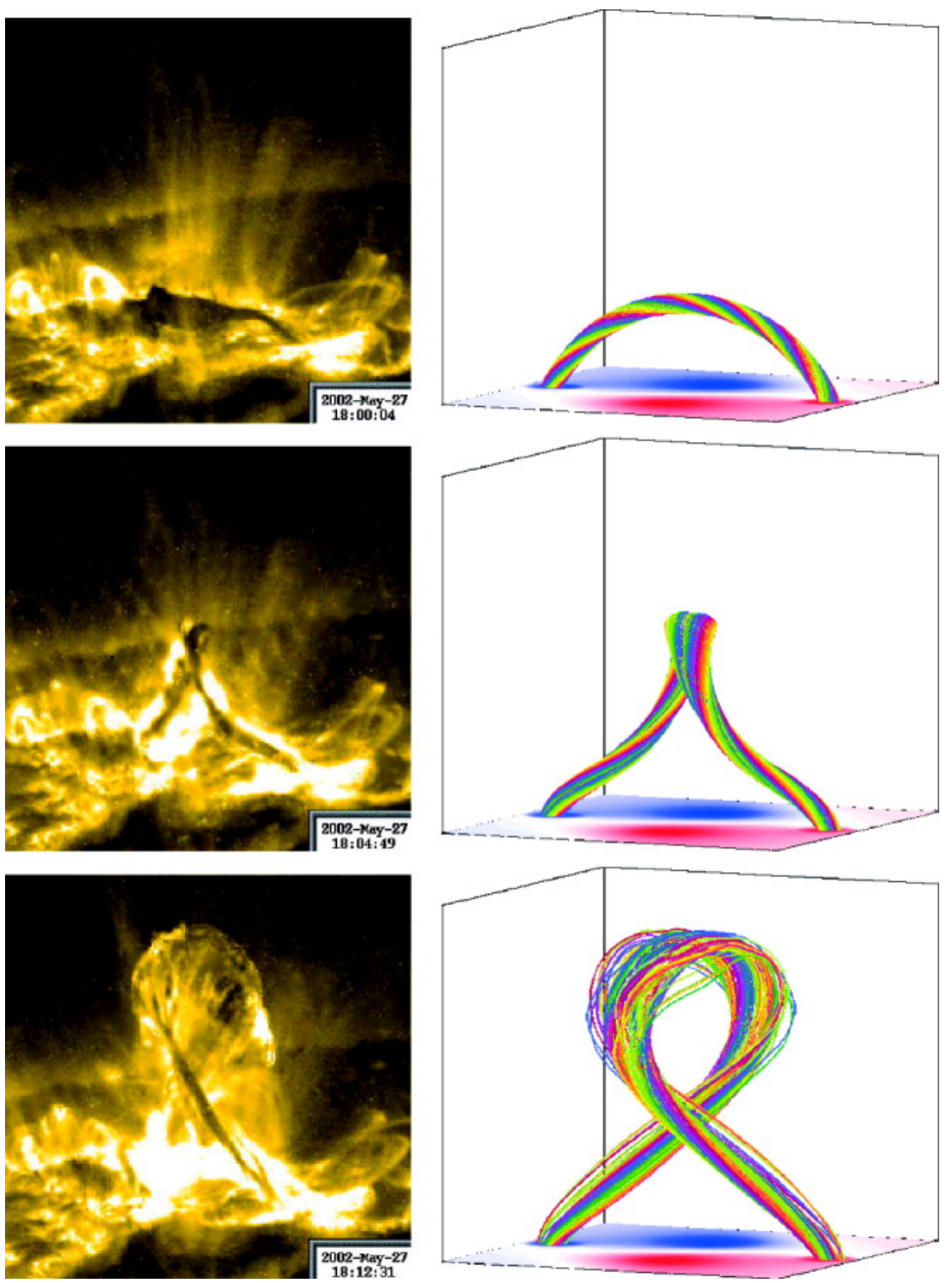

Fig. 4 Left: TRACE $195 \AA \AA$ images of the confined filament eruption on 2002 May 27. Right: Magnetic field lines outlining the core of the kink-unstable flux rope from the simulation of [82]. Figure from [82] reproduced by permission of the AAS.

cancelation at the PIL due to photospheric diffusion (e.g. [4, 5, 12]). The last flux transport process is important for the the formation of quiescent filament channels in decaying active regions (see [63]). Dynamic MHD simulations of the emergence of a twisted flux tube from the solar convection zone through the photosphere into 
the solar atmosphere as described in Section 2 have shown that shearing motions at the PIL and twisting motions of sunspots are naturally driven during the flux emergence, which transport twist from the interior into the corona. Furthermore, a vertical current sheet tends to develop underlying the emerged field, and the associated "tether-cutting" reconnections in the current sheet contribute to the buildup of the coronal flux rope and allow it to rise into the corona (Section 2). This picture of current sheet formation and magnetic reconnections contributing to the buildup of a coronal flux rope during the quasi-static phase has also been found in coronal MHD simulations of flux rope eruption (e.g. [12, 31, 32]). [12] performed a coronal MHD simulation of an initially potential, asymmetric bipolar field, which evolves as it is driven at the lower boundary by sub-Alfvénic, line-tied shearing motions and a slow magnetic diffusion that causes flux cancellation at the PIL. It is found that flux cancellation at the PIL transforms sheared arcades into a slowly rising and stable flux rope. Later a quasi-separatrix layer (QSL see more discussion below) topology develops with the formation of a vertical current sheet along the QSL and "tether-cutting" reconnections allow the flux rope to continue to rise slowly. As the flux rope reaches the height at which the decay index of the potential magnetic field reaches about 1.5, the flux rope undergoes a rapid acceleration. The conclusion from this study is that the non-ideal resistive processes of photospheric flux cancellation and tether-cutting coronal reconnections are not the trigger of eruption but are the key pre-eruptive mechanisms for the build up and rise of the coronal flux rope to the critical height at which the ideal torus instability causes the eruption.

Similar evolution for the build up of torus-unstable coronal flux ropes is found in the simulations of [31, 32]. In [31] a set of simulations are carried out where the coronal evolution is driven at the lower boundary by the slow emergence of a twisted flux rope into a pre-existing coronal arcade field, with a varying amount of twist being transported into the corona for the different cases before the emergence is stopped. In all the cases the emerged flux rope settles into a quasi-equilibrium after the emergence is stopped. Subsequently, the flux rope continues to rise quasistatically due to the "tether-cutting" reconnection in the vertical current sheet that forms underlying the flux rope (the two left panels in the top row of Figure 5), even though no more Poynting flux or helicity flux is transported into the corona and the coronal magnetic energy is slowly declining (3rd row panel in Figure 5). The flux rope is found to transition to a dynamic eruption (top right panel in Figure 5) with rapid acceleration and sharp release of the magnetic energy at varying times for the different cases (the 2nd and 3rd-row panels of Figure 5, but all corresponding to when roughly the same critical height is reached (bottom panel of Figure 5) where the decay index of the potential field reaches about 1.7. This nearly uniform height dependence for the onset of eruption suggests that the trigger of the eruption is caused by the onset of the torus instability, while the tether-cutting reconnection is contributing to the build-up of the flux rope and facilitating its (quasi-static) rise to the critical height for the onset of the instability. [32] further showed that the thermal signatures of such "tether-cutting" reconnections is the formation of a hot central low-density channel containing reconnected, twisted flux threading under the flux rope axis and on top of the central vertical current sheet. When viewed in the 

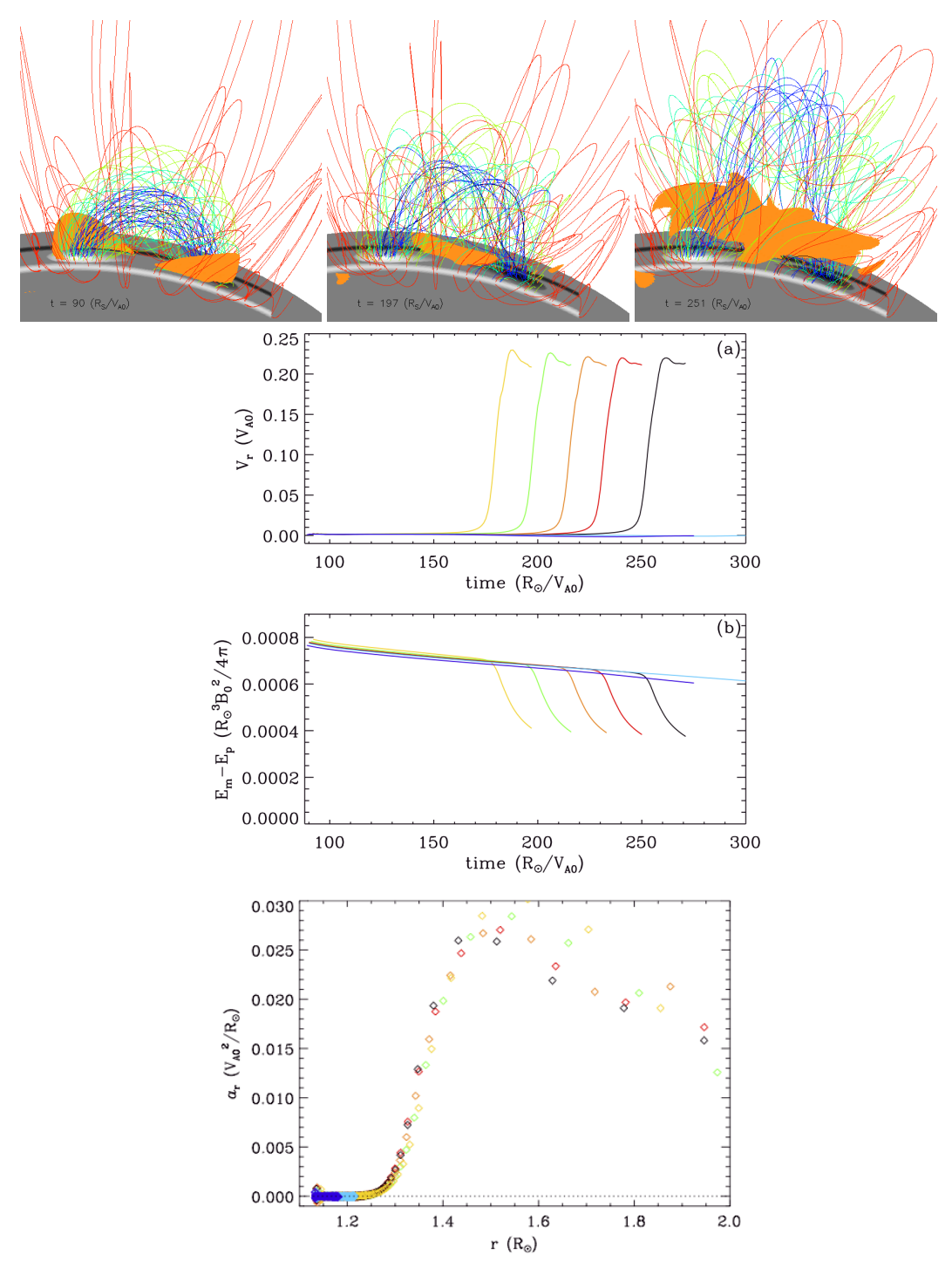

Fig. 5 Simulations by [31] of the buildup and eruption of torus-unstable coronal flux ropes. Top row panels: snapshots of 3D coronal flux rope with underlying current sheet formation (orange isosurfaces) during the quasi-static rise phase (two left panels) and at the onset of dynamic eruption when the critical height for the onset of the torus instability is reached (right panel). The evolution corresponds to the black curves in the lower panels. 2nd and 3rd-row panels show respectively the flux rope rise speed and the free magnetic energy as a function of time after the emergence is stopped, for the different cases (different colored curves) where different amount of twist is transported into the corona during the imposed flux emergence. Bottom panel: the acceleration of the flux rope as a function of the apex height for the different cases. Rapid acceleration takes place when roughly the same critical height is reached. Figure reproduced by permision of the AAS. 
line of sight roughly aligned with the hot channel (see Figure 6), the central vertical current sheet appears as a high-density vertical column with upward extensions as a U-shaped dense shell enclosing a central hot, low-density void corresponding to the central hot channel. Such thermal features may correspond to the observed substructures (central hot cavity on top of prominence "horns") that have been observed within coronal prominence cavities (see [42]).
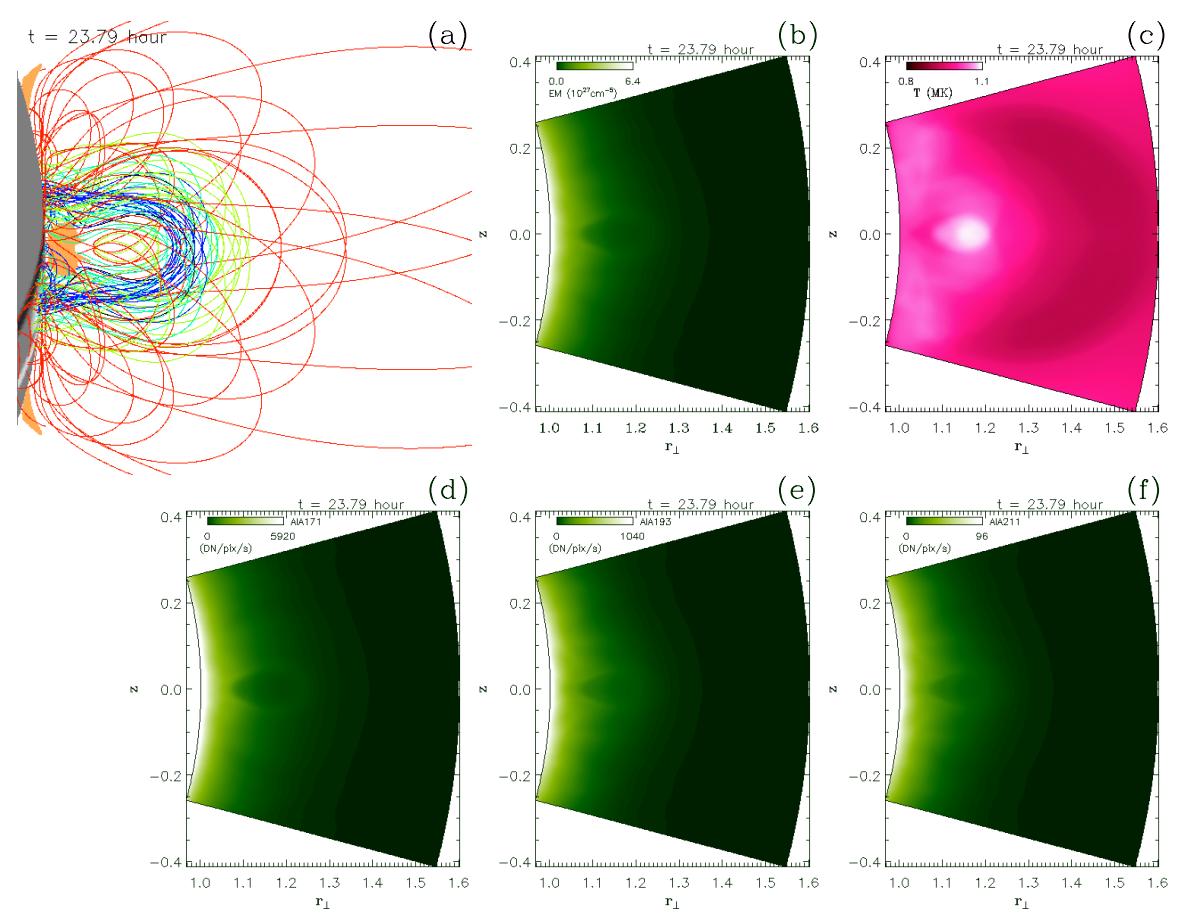

Fig. 6 From [32]. (a) 3D flux rope field lines and the underlying current sheet (orange iso-surface of $J / B$ ) viewed above the limb along the line of sight that is roughly aligned with the hot channel. The hot channel forms due to the accumulation of the reconnected flux rising into the flux rope produced by the "tether-cutting" reconnections during the quasi-static rise phase. (b) and (c) show respectively the modeled emission measure and line-of-sight averaged temperature showing the central hot low density cavity caused by the hot channel, and (d), (e), and (f) show respectively the synthetic SDO/AIA intensity images at $171 \AA, 193 \AA$, and $211 \AA$, as viewed from the same line of sight. Figure from [32] reproduced by permission of the AAS.

The central vertical current sheet underlying the simulated flux rope (Figure 6/a)) is found to have formed along topological structures identified as quasi separatrix layers (QSLs, see Figure 7), which are regions of the magnetic volume where the field line connectivity to the line-tied surface experiences drastic variations ([27, 28, 81]). They are identified by estimating the so-called squashing degree, $Q$, which measures the "squashing" of an elementary flux tube cross-section as it is mapped from one foot point to the other $([81,79$, 72] $)$. QSLs correspond to re- 

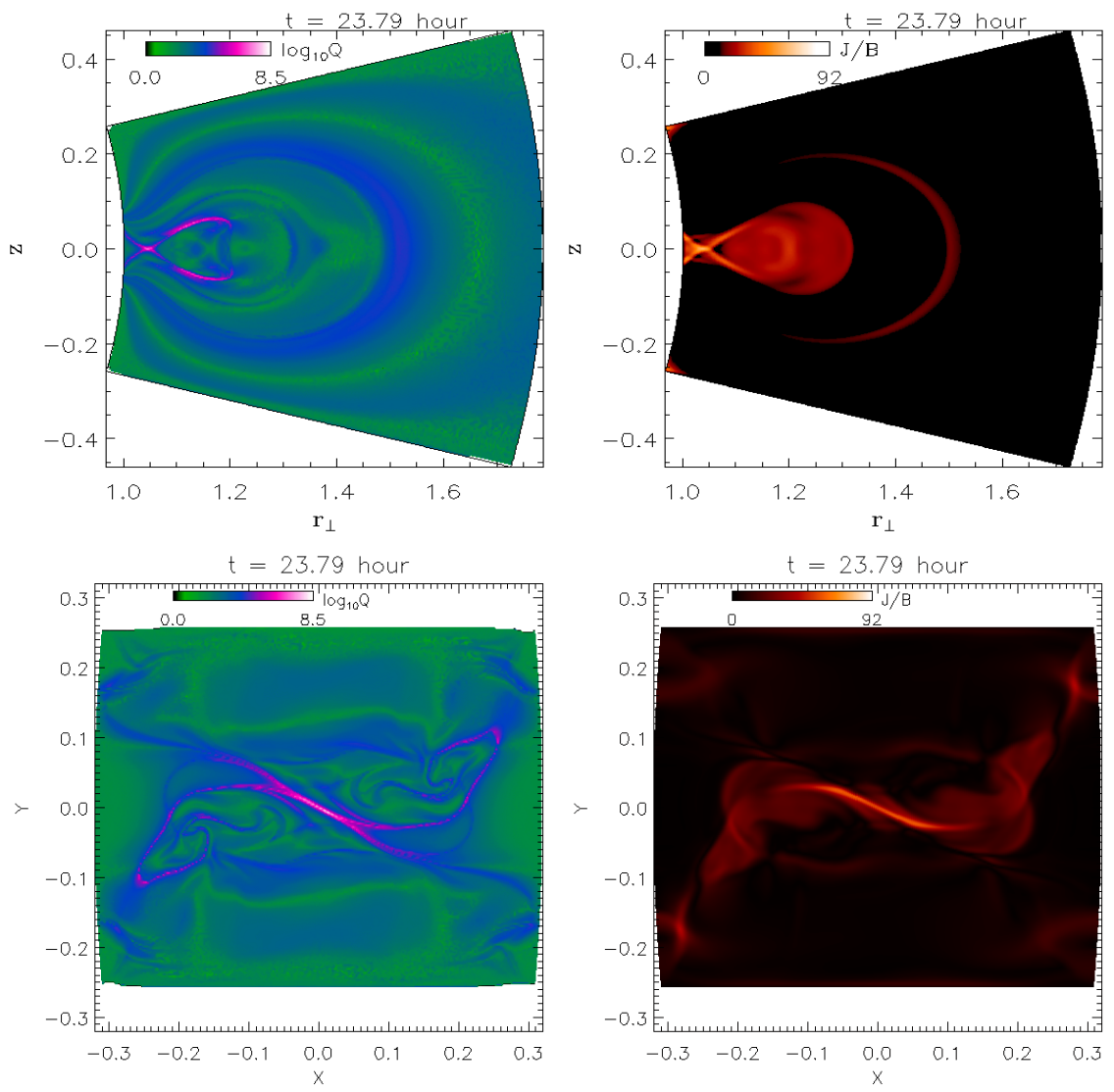

Fig. 7 From the simulation of [32]. Squashing degree $Q$ in respectively the central meridional cross-section of the flux rope (upper left panel) and a horizontal cross-section below the hot channel of the flux rope (lower left panel). The high Q value outlines the location of the QSLs, along which intense current sheets form as shown in the corresponding cross-sections of $J / B$ in the right panels. Figure from $[32]$ reproduced by permission of the AAS.

gions of very large $\mathrm{Q}$. They are a generalization of the concept of separatrices at which the field line linkage is discontinuous. Similar to a separatrix, a QSL divides the coronal domain into quasi-connectivity domains, and due to the drastic change of the field line connectivity at the QSL, it is a site along which current sheets or magnetic tangential discontinuities tend to form and where magnetic reconnections take place (e.g. [28, 11, 76]). The QSL with the highest Q values shown in the central meridional cross-section of the simulated flux rope (top left panel of Figure 7) correspond to the mid cross-section of the so-called Hyperbolic Flux Tube (HFT), a generalization of the $\mathrm{X}$-line configuration, which divides the magnetic volume into four distinct domains of magnetic field connectivity (e.g. [79, 11, 76]). The central vertical current sheet underlying the flux rope forms along the HFT (see the top 
right panel of Figure 77. [32] suggests that the observed density feature of a dense prominence column with upward extending "horns" (see [42]) corresponds to the current sheet that forms along the HFT.

As has been discussed above, magnetic reconnection may be playing an important role for the build up of an unstable coronal flux rope before eruption. It has also been shown that magnetic reconnection is critically important for producing an ejective eruption of the magnetic flux in a CME. The energetics for the magnetic storage models of CMEs requires that the magnetic energy of the pre-eruption field exceeds the subsequent magnetic energy during and after the eruption. If the eruption process remains ideal as the flux rope is moving out to infinity, it would have to stretch out its overlying fields to infinity. This may be energetically impossible since it has been shown that for a given normal magnetic flux distribution at the photosphere, the energy of any three-dimensional force free magnetic field with all the field lines simply connected to the photosphere is smaller than the energy of the corresponding fully open magnetic field $([1,2,78])$. This is the so-called AlySturrock energy constraint. Thus there is not enough energy in a pre-eruption force free field to stretch all the field lines to infinity to reach a fully open field. One way to get around this difficulty is that an ideal MHD instability or loss of equilibrium can extend the field lines to some height while driving the formation of a current sheet behind the erupting flux rope, and a fast reconnection in the driven current sheet allows the stretched-out field lines to successively reconnect and close back down behind the flux rope as the flux rope is moving out (e.g. [74]). Thus a fast magnetic reconnection is necessary to sustain the eruption and allow the flux rope to escape to infinity.

\subsection{Sudden onset of fast magnetic reconnection as trigger for eruptions}

Besides the ideal MHD instability and loss of equilibrium processes, another likely trigger for the onset of eruption is the sudden onset or enhancement of fast magnetic reconnection in a current sheet. Detailed theoretical and computational studies of magnetic reconnection have demonstrated that such sudden onset behavior of fast magnetic reconnection can be achieved through "collisionless" effects when the current sheet thickness falls to the order of the ion inertial length (e.g. [17, 21]), or even in the resistive MHD regime due to the onset of the plasmoid instability for extended thin current sheets of high Lundquist number (e.g. [18]). In the following example MHD simulations that focus on studying the large scale dynamic evolution of eruptions triggered by the onset of fast magnetic reconnection, the reconnection processes are modeled with resistive MHD using a variable magnetic diffusion $\eta$ or a scale dependent numerical diffusivity.

One of the early simulations that clearly demonstrated resistivity triggered eruption is [68], in which an axisymmetric dipolar coronal arcade field outside the solar surface is subject to a slow shearing motion at its footpoints (see Figure 87. 
The initial magnetic field is a Sun-centered dipole field $(t=0$ panel), which in-
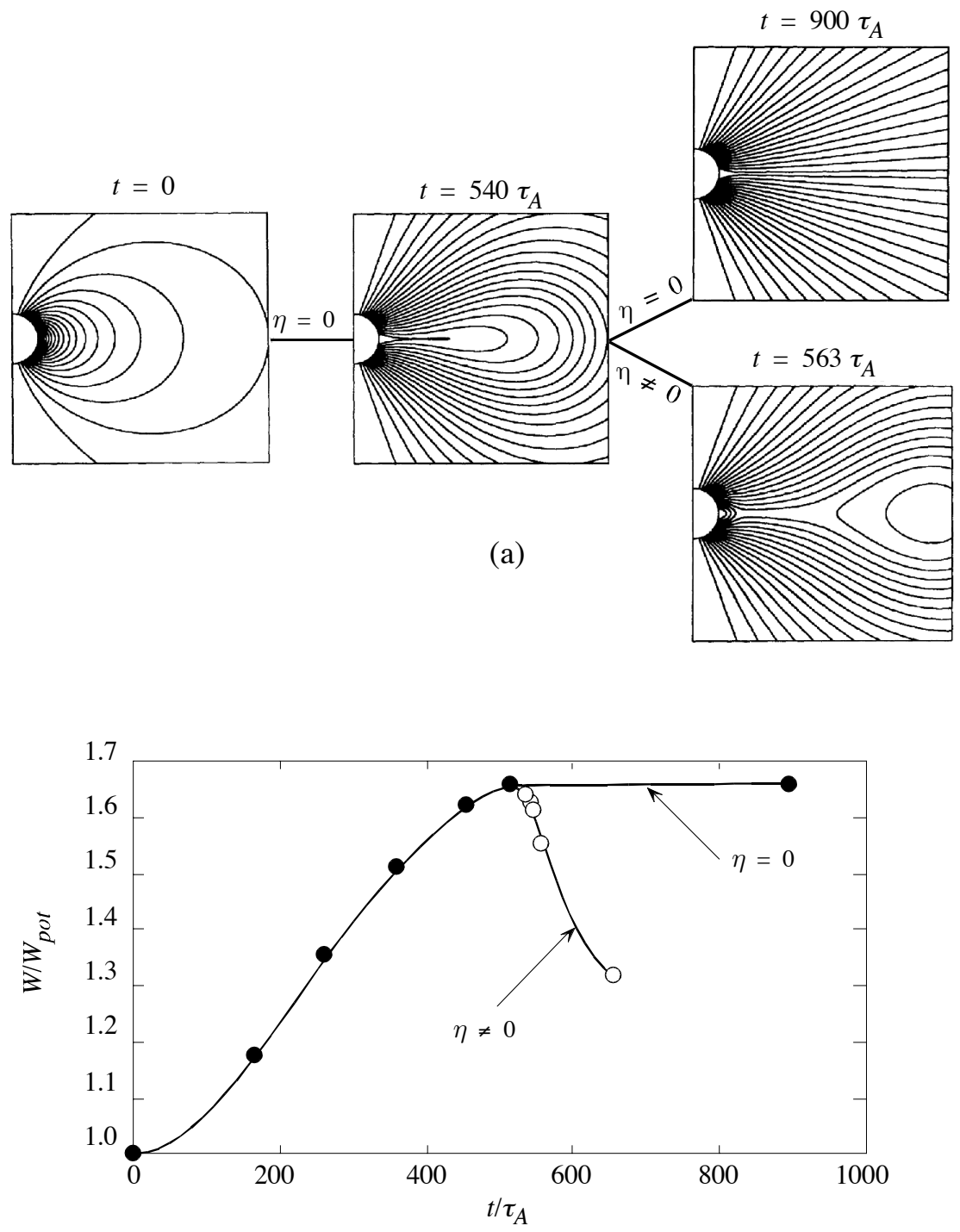

(b)

Fig. 8 Simulation from [68] of an axisymmetric coronal arcade field subject to slow shearing motion at its footpoints on the solar surface with the two hemispheres moving in opposite directions. The panels in (a) show snapshots of the magnetic field evolution with $\eta$ remaining zero .vs. being instantaneously switched on after $t=540 \tau_{A}$. Panel (b) shows the evolution of the magnetic energy for the two different cases. Figure reproduced from the review [39] with permission. 
flates quasi-statically through a sequence of force free equilibria $\left(t=540 \tau_{A}\right.$ and $t=900 \tau_{A}$ panels, where $\tau_{A}$ is the Alfvén time defined as the solar radius divided by the Alfvén speed) with increasing magnetic energy asymptotically towards a fully open field of the maximum energy (solid black dots in panel (b)) if the evolution remains ideal with the magnetic diffusivity $\eta=0$. For such two-dimensional force free configurations with translational symmetry, shearing causes the arcade to expand quasi-statically outwards towards a fully opened state without developing an ideal loss of equilibrium. However, it is found that during the quasi-static expansion if $\eta$ is instantaneously increased to a value which gives an effective magnetic Reynolds number of about $10^{4}$, rapid reconnection takes place at the current sheet at the equator, leading to the formation of a plasmoid which is ejected dynamically (see the $t=563 \tau_{A}$ panel), and a sharp release of the magnetic energy (see the white circles in panel (b)).

Another well studied model for CME initiation triggered by magnetic reconnection is the "breakout" model first described by [6]. MHD simulations based on the breakout model have been carried out in both 2.5D and 3D configurations (e.g. [65, 62]), and in a 3D configuration with an ambient solar wind (e.g. [85]). [50] carried out 2.5D simulations of the breakout model with adaptive mesh refinement, achieving high resolutions in the current sheet in global scale dynamic simulations of eruption, and thus allowing identification of the various key phases of the reconnections in relation to the eruption. The basics of how the breakout model works is described as follows (see Figure 9). The initial potential magnetic field outside the

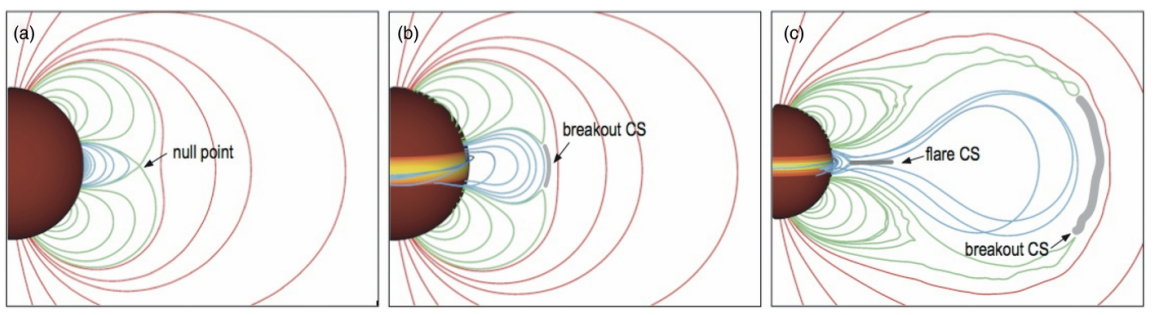

Fig. 9 Key steps in the simulation of [50] illustrating the basic working and structure (including current sheets CS) in the break out model. Figure reproduced from [50] with permission from the AAS.

solar surface contains a multiflux system with three neutral lines on the surface and four distinct flux systems (Figure 9 a)): a central arcade straddling the equator (blue field lines), two side arcades associated with the neutral lines at mid latitudes (green field lines), and a polar flux system overlying the three arcades (red lines). There are two separatrix surfaces that define the boundaries between the various flux systems, and a null point above the central arcade. In the initial quasi-static phase, the inner arcade field of the central arcade system is being sheared slowly to become the filament channel (sheared field) and remains confined by the unsheared arcade to build up free magnetic energy. As long as the reconnection between the unsheared (blue) arcade of the central system and the overlying red arcade is slow compared to the 
shearing, i.e. nearly ideal, energy is built up. However the current sheet formed at the null point eventually becomes thin enough (see the breakout CS in Figure 9 (b)) and the scale-dependent numerical resistivity causes a sudden onset of the fast magnetic reconnection in the breakout CS (see Figure 10 (b)). The fast breakout reconnection

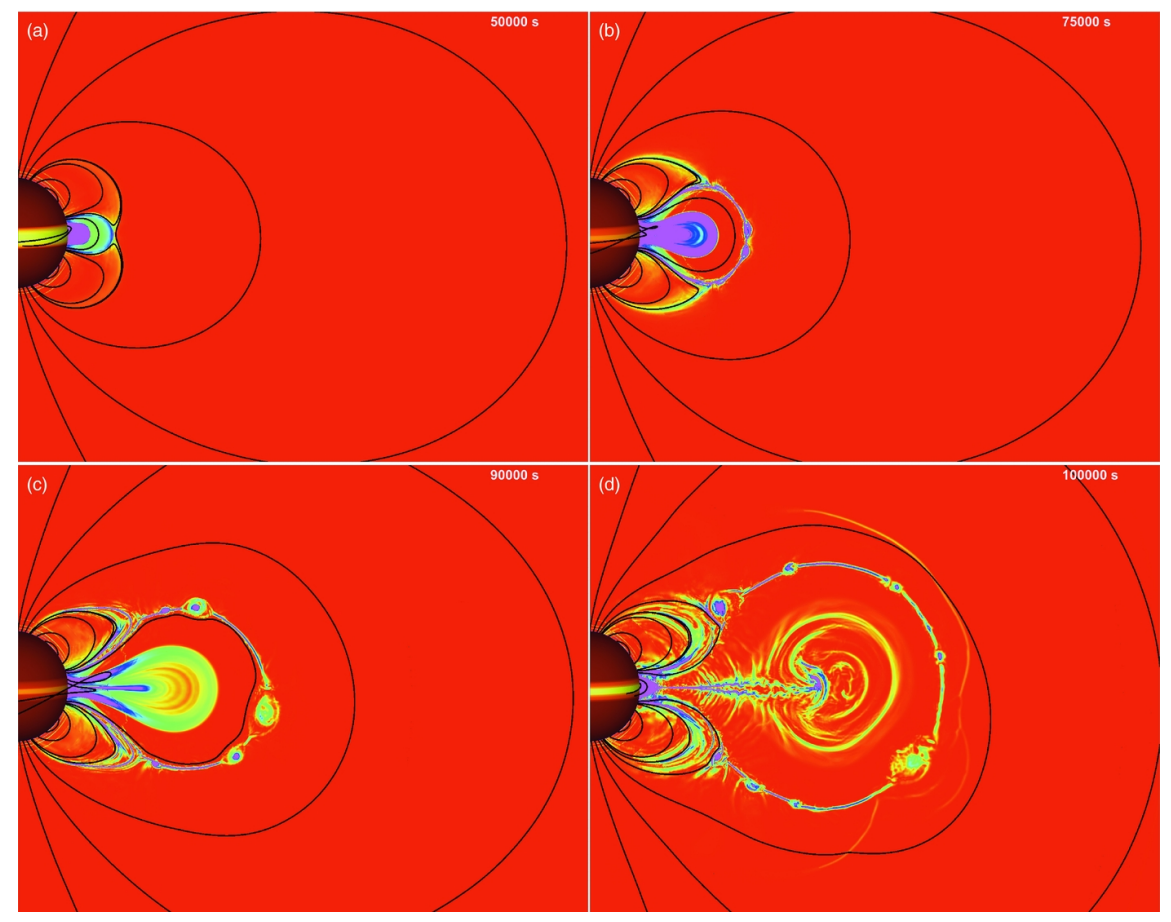

Fig. $10 B_{\phi}$ on the solar surface, and normalized current density and magnetic field lines in the meridional cross-section, at selected times in the breakout simulation of [50]. Figure from [50] reproduced by permission of the AAS.

rapidly transfers the confining flux in the unsheared arcade and the overlying outer arcade into the side lobes, causing an outward expansion of the filament channel field and the confinement is found to be permanently lost. Thus the onset of the breakout reconnection is the trigger of the CME and if it were to continue to act alone (Figure 10(c)), it would have led to a complete but slow outward expansion of the filament channel field with a vertical current sheet extending downward to low heights in the corona, and thus with most of the free energy retained. But then a sudden onset of fast reconnection in the flare current sheet is found to trigger impulsive energy release with the formation of a flux rope that accelerates impulsively (Figure 9 (c) and 10(d)). Thus in this model, the resistive process of the onset of fast magnetic reconnection is the initiation mechanism (or trigger) for both the CME onset (loss of equilibrium for the filament channel field) as well as the later onset 
of impulsive CME acceleration. For the former it is the onset of the fast breakout reconnection and for the latter it is the onset of fast reconnection in the flare CS. The question then is whether it is possible to observationally identify these two stages of the development.

\section{Concluding remarks}

The question of how twisted and sheared coronal magnetic structures capable of driving filament eruptions form is examined with focus on emerging active regions. MHD simulations of flux emergence have shown that many commonly observed features associated with flare and CME productive active regions, such as magnetic and velocity shear at the PILs, rotating sunspots, sigmoid-shaped X-ray loops and filaments, can be explained by the emergence of a twisted magnetic flux tube from the solar interior into the atmosphere. It is found that shear and twisting motions driven by the Lorentz force of the emerging tube are the major means (instead of direct vertical emergence through the photosphere) by which magnetic helicity (twist) and energy are transported into the corona. It is also found that current sheet formation and the associated tether-cutting reconnections are important for the buildup of the coronal flux rope and allow it to rise into the corona. This is found in both simulations of flux emergence through the photosphere as well as simulations of line-tied coronal flux ropes evolving quasi-statically towards the onset of eruption.

Several basic mechanisms that can trigger the sudden disruption of the quasiequilibrium coronal magnetic structures and explosive release of the stored free magnetic energy are discussed. These include both ideal processes such as the onset of the helical kink instability and the torus instability of a coronal flux rope, and the non-ideal processes of the onset of fast magnetic reconnections in a current sheet. It is found that even when the triggering mechanism is an ideal instability or loss of equilibrium of a coronal flux rope, magnetic reconnection is playing an important role in its buildup during the quasi-static stage, as well as for sustaining the eruption and allowing for an ejection of flux rope due to the Aly-Sturrock energy constraint. MHD simulations have shown that such "hybrid" models of the quasi-static buildup of torus unstable coronal flux ropes with the development of the "HFT" topology can explain some of the thermal features observed to develop in pre-eruption coronal prominence cavities. MHD simulations of the non-linear evolution of kink-unstable coronal flux ropes have also shown magnetic field evolution that resembles remarkably well the observed morphology of some of the highly

writhing filament eruptions. Fast magnetic reconnection can also trigger eruptions as described in the breakout model without the presence of a more twisted pre-eruption coronal flux rope capable of developing ideal instabilities. Simulations based on this model show that the onset of fast reconnection in the flare current sheet that rapidly creates a highly twisted flux rope is needed for the onset of the impulsive acceleration of the CMEs. 
It should be noted that the discussion of this chapter has focused entirely on nearly force free coronal structures, ignoring the non force free effects of the prominence weight for example. There is an important body of work on the role of prominence mass in energy storage of the prominence magnetic field and CME energetics (e.g. [60, 61, 38, 59, 89, 90]), especially with regard to overcoming the Aly-Sturrock energy constraint.

Acknowledgements The author would like to thank Fang Fang, Terry Forbes, Judy Karpen, Zoran Mikic, Slava Titov, and Tibor Török for granting permission to reprint Figures from their original publications. The National Center for Atmospheric Research is sponsored by the National Science Foundation. 


\section{References}

1. Aly, J.J.: On some properties of force-free magnetic fields in infinite regions of space. ApJ 283, 349-362 (1984). DOI 10.1086/162313

2. Aly, J.J.: How much energy can be stored in a three-dimensional force-free magnetic field? ApJ 375, L61-L64 (1991). DOI 10.1086/186088

3. Amari, T., Luciani, J.F., Aly, J.J.: Coronal Magnetohydrodynamic Evolution Driven by Subphotospheric Conditions. ApJ 615, L165-L168 (2004). DOI 10.1086/426317

4. Amari, T., Luciani, J.F., Aly, J.J., Mikic, Z., Linker, J.: Coronal Mass Ejection: Initiation, Magnetic Helicity, and Flux Ropes. I. Boundary Motion-driven Evolution. ApJ 585, 10731086 (2003). DOI 10.1086/345501

5. Amari, T., Luciani, J.F., Aly, J.J., Mikic, Z., Linker, J.: Coronal Mass Ejection: Initiation, Magnetic Helicity, and Flux Ropes. II. Turbulent Diffusion-driven Evolution. ApJ 595, 12311250 (2003). DOI 10.1086/377444

6. Antiochos, S.K., DeVore, C.R., Klimchuk, J.A.: A Model for Solar Coronal Mass Ejections. ApJ 510, 485-493 (1999). DOI 10.1086/306563

7. Anzer, U.: The Stability of Force-Free Magnetic Fields with Cylindrical Symmetry in the Context of Solar Flares. Sol. Phys. 3, 298-315 (1968). DOI 10.1007/BF00155164

8. Archontis, V., Hood, A.W.: Magnetic flux emergence: a precursor of solar plasma expulsion. Astron. Astrophys. 537, A62 (2012). DOI 10.1051/0004-6361/201116956

9. Archontis, V., Hood, A.W., Savcheva, A., Golub, L., Deluca, E.: On the Structure and Evolution of Complexity in Sigmoids: A Flux Emergence Model. ApJ 691, 1276-1291 (2009). DOI 10.1088/0004-637X/691/2/1276

10. Archontis, V., Hood, A.W., Tsinganos, K.: Recurrent Explosive Eruptions and the "Sigmoidto-arcade" Transformation in the Sun Driven by Dynamical Magnetic Flux Emergence. ApJ 786, L21 (2014). DOI 10.1088/2041-8205/786/2/L21

11. Aulanier, G., Pariat, E., Démoulin, P.: Current sheet formation in quasi-separatrix layers and hyperbolic flux tubes. Astron. Astrophys. 444, 961-976 (2005). DOI 10.1051/0004-6361: 20053600

12. Aulanier, G., Török, T., Démoulin, P., DeLuca, E.E.: Formation of Torus-Unstable Flux Ropes and Electric Currents in Erupting Sigmoids. ApJ 708, 314-333 (2010). DOI 10.1088/0004-637X/708/1/314

13. Bateman, B.: MHD Instabilities. MIT, Cambridge, MA (1978)

14. Berger, M.A.: Rigorous new limits on magnetic helicity dissipation in the solar corona. Geophysical and Astrophysical Fluid Dynamics 30, 79-104 (1984). DOI 10.1080/ 03091928408210078

15. Berger, M.A., Field, G.B.: The topological properties of magnetic helicity. Journal of Fluid Mechanics 147, 133-148 (1984). DOI 10.1017/S0022112084002019

16. Berger, T., Testa, P., Hillier, A., Boerner, P., Low, B.C., Shibata, K., Schrijver, C., Tarbell, T., Title, A.: Magneto-thermal convection in solar prominences. Nature 472, 197-200 (2011). DOI 10.1038/nature09925

17. Bhattacharjee, A.: Impulsive Magnetic Reconnection in the Earth's Magnetotail and the Solar Corona. Annu. Rev. Astron. Astrophys. 42, 365-384 (2004). DOI 10.1146/annurev.astro.42. 053102.134039

18. Bhattacharjee, A., Huang, Y.M., Yang, H., Rogers, B.: Fast reconnection in high-Lundquistnumber plasmas due to the plasmoid Instability. Physics of Plasmas 16(11), 112,102 (2009). DOI 10.1063/1.3264103

19. Brown, D.S., Nightingale, R.W., Alexander, D., Schrijver, C.J., Metcalf, T.R., Shine, R.A., Title, A.M., Wolfson, C.J.: Observations of Rotating Sunspots from TRACE. Sol. Phys. 216, 79-108 (2003). DOI 10.1023/A:1026138413791

20. Canou, A., Amari, T., Bommier, V., Schmieder, B., Aulanier, G., Li, H.: Evidence for a PreEruptive Twisted Flux Rope Using the Themis Vector Magnetograph. ApJL 693, L27-L30 (2009). DOI 10.1088/0004-637X/693/1/L27 
21. Cassak, P.A., Shay, M.A., Drake, J.F.: Catastrophe Model for Fast Magnetic Reconnection Onset. Physical Review Letters 95(23), 235002 (2005). DOI 10.1103/PhysRevLett.95.235002

22. Chae, J., Wang, H., Qiu, J., Goode, P.R., Strous, L., Yun, H.S.: The Formation of a Prominence in Active Region NOAA 8668. I. SOHO/MDI Observations of Magnetic Field Evolution. ApJ 560, 476-489 (2001). DOI 10.1086/322491

23. Chatterjee, P., Fan, Y.: Simulation of Homologous and Cannibalistic Coronal Mass Ejections produced by the Emergence of a Twisted Flux Rope into the Solar Corona. ApJ 778, L8 (2013). DOI 10.1088/2041-8205/778/1/L8

24. de Toma, G., Casini, R., Burkepile, J.T., Low, B.C.: Rise of a Dark Bubble through a Quiescent Prominence. ApJL 687, L123-L126 (2008). DOI 10.1086/593326

25. Démoulin, P.: Recent theoretical and observational developments in magnetic helicity studies. Advances in Space Research 39, 1674-1693 (2007). DOI 10.1016/j.asr.2006.12.037

26. Démoulin, P., Aulanier, G.: Criteria for Flux Rope Eruption: Non-equilibrium Versus Torus Instability. ApJ 718, 1388-1399 (2010). DOI 10.1088/0004-637X/718/2/1388

27. Démoulin, P., Henoux, J.C., Priest, E.R., Mandrini, C.H.: Quasi-Separatrix layers in solar flares. I. Method. Astron. Astrophys. 308, 643-655 (1996)

28. Démoulin, P., Priest, E.R., Lonie, D.P.: Three-dimensional magnetic reconnection without null points 2. Application to twisted flux tubes. Journal of Geophysical Research (Space Physics) 101, 7631-7646 (1996). DOI 10.1029/95JA03558

29. Engvold, O.: Chapter 2: Description of prominences, including classification. In: J.C. Vial, O. Engvold (eds.) Solar Prominences. Springer (2014)

30. Fan, Y.: The Emergence of a Twisted Flux Tube into the Solar Atmosphere: Sunspot Rotations and the Formation of a Coronal Flux Rope. ApJ 697, 1529-1542 (2009). DOI 10.1088/ 0004-637X/697/2/1529

31. Fan, Y.: On the Eruption of Coronal Flux Ropes. ApJ 719, 728-736 (2010). DOI 10.1088/ 0004-637X/719/1/728

32. Fan, Y.: Thermal Signatures of Tether-cutting Reconnections in Pre-eruption Coronal Flux Ropes: Hot Central Voids in Coronal Cavities. ApJ 758, 60 (2012). DOI 10.1088/0004-637X/ $758 / 1 / 60$

33. Fan, Y., Gibson, S.E.: Numerical Simulations of Three-dimensional Coronal Magnetic Fields Resulting from the Emergence of Twisted Magnetic Flux Tubes. ApJ 609, 1123-1133 (2004)

34. Fan, Y., Gibson, S.E.: Onset of Coronal Mass Ejections Due to Loss of Confinement of Coronal Flux Ropes. ApJ 668, 1232-1245 (2007). DOI 10.1086/521335

35. Fang, F., Manchester, W., Abbett, W.P., van der Holst, B.: Simulation of Flux Emergence from the Convection Zone to the Corona. ApJ 714, 1649-1657 (2010). DOI 10.1088/0004-637X/ $714 / 2 / 1649$

36. Fang, F., Manchester IV, W., Abbett, W.P., van der Holst, B.: Buildup of Magnetic Shear and Free Energy during Flux Emergence and Cancellation. ApJ 754, 15 (2012). DOI 10.1088/ 0004-637X/754/1/15

37. Fang, F., Manchester IV, W., Abbett, W.P., van der Holst, B.: Dynamic Coupling of Convective Flows and Magnetic Field during Flux Emergence. ApJ 745, 37 (2012). DOI 10.1088/0004-637X/745/1/37

38. Fong, B., Low, B.C., Fan, Y.: Quiescent Solar Prominences and Magnetic-Energy Storage. ApJ 571, 987-998 (2002). DOI 10.1086/340070

39. Forbes, T.G.: A review on the genesis of coronal mass ejections. Journal of Geophysical Research (Space Physics) 105, 23,153-23,166 (2000). DOI 10.1029/2000JA000005

40. Forbes, T.G., Linker, J.A., Chen, J., Cid, C., Kóta, J., Lee, M.A., Mann, G., Mikić, Z., Potgieter, M.S., Schmidt, J.M., Siscoe, G.L., Vainio, R., Antiochos, S.K., Riley, P.: CME Theory and Models, in Coronal Mass Ejections, Space Sciences Series of ISSI, Volume 21. ISBN 978-0-387-45086-5. Springer, p. 251 (2006)

41. Forbes, T.G., Priest, E.R.: Photospheric Magnetic Field Evolution and Eruptive Flares. ApJ 446, 377 (1995). DOI 10.1086/175797

42. Gibson, S.E.: Chapter 13: Coronal cavities: observations and implications for the magnetic environment of prominences. In: J.C. Vial, O. Engvold (eds.) Solar Prominences. Springer (2014) 
43. Gibson, S.E., Fan, Y.: Coronal prominence structure and dynamics: A magnetic flux rope interpretation. Journal of Geophysical Research (Space Physics) 111, A12103 (2006). DOI 10.1029/2006JA011871

44. Gibson, S.E., Fletcher, L., Del Zanna, G., Pike, C.D., Mason, H.E., Mandrini, C.H., Démoulin, P., Gilbert, H., Burkepile, J., Holzer, T., Alexander, D., Liu, Y., Nitta, N., Qiu, J., Schmieder, B., Thompson, B.J.: The Structure and Evolution of a Sigmoidal Active Region. ApJ 574, 1021-1038 (2002). DOI 10.1086/341090

45. Gopalswamy, N., Shimojo, M., Lu, W., Yashiro, S., Shibasaki, K., Howard, R.A.: Prominence Eruptions and Coronal Mass Ejection: A Statistical Study Using Microwave Observations. ApJ 586, 562-578 (2003). DOI 10.1086/367614

46. Hood, A.W., Priest, E.R.: Critical conditions for magnetic instabilities in force-free coronal loops. Geophysical and Astrophysical Fluid Dynamics 17, 297-318 (1981). DOI 10.1080/ 03091928108243687

47. Isenberg, P.A., Forbes, T.G.: A Three-dimensional Line-tied Magnetic Field Model for Solar Eruptions. ApJ 670, 1453-1466 (2007). DOI 10.1086/522025

48. Jiang, Y., Zheng, R., Yang, J., Hong, J., Yi, B., Yang, D.: Rapid Sunspot Rotation Associated with the X2.2 Flare on 2011 February 15. ApJ 744, 50 (2012). DOI 10.1088/0004-637X/744/ $1 / 50$

49. Karpen, J.: Chapter 10: Plasma structure and dynamics. In: J.C. Vial, O. Engvold (eds.) Solar Prominences. Springer (2014)

50. Karpen, J.T., Antiochos, S.K., DeVore, C.R.: The Mechanisms for the Onset and Explosive Eruption of Coronal Mass Ejections and Eruptive Flares. ApJ 760, 81 (2012). DOI 10.1088/ 0004-637X/760/1/81

51. Kippenhahn, R., Schlüter, A.: Eine Theorie der solaren Filamente. Mit 7 Textabbildungen. Zeitschrift für Astrophysik 43, 36 (1957)

52. Kliem, B., Török, T.: Torus Instability. Physical Review Letters 96(25), 255002 (2006). DOI 10.1103/PhysRevLett.96.255002

53. Leake, J.E., Linton, M.G., Török, T.: Simulations of Emerging Magnetic Flux. I. The Formation of Stable Coronal Flux Ropes. ApJ 778, 99 (2013). DOI 10.1088/0004-637X/778/2/99

54. Lin, J., Forbes, T.G., Isenberg, P.A., Démoulin, P.: The Effect of Curvature on Flux-Rope Models of Coronal Mass Ejections. ApJ 504, 1006-1019 (1998). DOI 10.1086/306108

55. Lites, B.W.: Magnetic Flux Ropes in the Solar Photosphere: The Vector Magnetic Field under Active Region Filaments. ApJ 622, 1275-1291 (2005). DOI 10.1086/428080

56. Liu, R., Alexander, D.: Hard X-ray Emission in Kinking Filaments. ApJ 697, 999-1009 (2009). DOI 10.1088/0004-637X/697/2/999

57. Liu, W., Berger, T.E., Low, B.C.: First SDO/AIA Observation of Solar Prominence Formation Following an Eruption: Magnetic Dips and Sustained Condensation and Drainage. ApJL 745, L21 (2012). DOI 10.1088/2041-8205/745/2/L21

58. Longcope, D.W., Welsch, B.T.: A Model for the Emergence of a Twisted Magnetic Flux Tube. ApJ 545, 1089-1100 (2000). DOI 10.1086/317846

59. Low, B.C., Fong, B., Fan, Y.: The Mass of a Solar Quiescent Prominence. ApJ 594, 10601067 (2003). DOI 10.1086/377042

60. Low, B.C., Smith, D.F.: The free energies of partially open coronal magnetic fields. ApJ 410, 412-425 (1993). DOI 10.1086/172758

61. Low, B.C., Zhang, M.: The Hydromagnetic Origin of the Two Dynamical Types of Solar Coronal Mass Ejections. ApJ 564, L53-L56 (2002). DOI 10.1086/338798

62. Lynch, B.J., Antiochos, S.K., DeVore, C.R., Luhmann, J.G., Zurbuchen, T.H.: Topological Evolution of a Fast Magnetic Breakout CME in Three Dimensions. ApJ 683, 1192-1206 (2008). DOI 10.1086/589738

63. Mackay, D.: Chapter 14: Formation and large-scale patterns of filament channels and filaments. In: J.C. Vial, O. Engvold (eds.) Solar Prominences. Springer (2014)

64. Mackay, D.H., van Ballegooijen, A.A.: New Results in Modeling the Hemispheric Pattern of Solar Filaments. ApJL 621, L77-L80 (2005). DOI 10.1086/428904 
65. MacNeice, P., Antiochos, S.K., Phillips, A., Spicer, D.S., DeVore, C.R., Olson, K.: A Numerical Study of the Breakout Model for Coronal Mass Ejection Initiation. ApJ 614, 1028-1041 (2004). DOI 10.1086/423887

66. Magara, T.: A Model for Dynamic Evolution of Emerging Magnetic Fields in the Sun. ApJ 605, 480-492 (2004)

67. Manchester IV, W., Gombosi, T., DeZeeuw, D., Fan, Y.: Eruption of a Buoyantly Emerging Magnetic Flux Rope. ApJ 610, 588-596 (2004). DOI 10.1086/421516

68. Mikic, Z., Linker, J.A.: Disruption of coronal magnetic field arcades. ApJ 430, 898-912 (1994). DOI 10.1086/174460

69. Moore, R.L., Sterling, A.C., Hudson, H.S., Lemen, J.R.: Onset of the Magnetic Explosion in Solar Flares and Coronal Mass Ejections. ApJ 552, 833-848 (2001). DOI 10.1086/320559

70. Munro, R.H., Gosling, J.T., Hildner, E., MacQueen, R.M., Poland, A.I., Ross, C.L.: The association of coronal mass ejection transients with other forms of solar activity. Sol. Phys. 61, 201-215 (1979). DOI 10.1007/BF00155456

71. Okamoto, T.J., Tsuneta, S., Lites, B.W., Kubo, M., Yokoyama, T., Berger, T.E., Ichimoto, K., Katsukawa, Y., Nagata, S., Shibata, K., Shimizu, T., Shine, R.A., Suematsu, Y., Tarbell, T.D., Title, A.M.: Emergence of a Helical Flux Rope under an Active Region Prominence. ApJL 673, L215-L218 (2008). DOI 10.1086/528792

72. Pariat, E., Démoulin, P.: Estimation of the squashing degree within a three-dimensional domain. Astron. Astrophys. 541, A78 (2012). DOI 10.1051/0004-6361/201118515

73. Petrie, G.J.D., Low, B.C.: The Dynamical Consequences of Spontaneous Current Sheets in Quiescent Prominences. ApJS 159, 288-313 (2005). DOI 10.1086/431149

74. Priest, E.R., Forbes, T.G.: The magnetic nature of solar flares. A\&A Rev. 10, 313-377 (2002). DOI $10.1007 / \mathrm{s} 001590100013$

75. Raadu, M.A.: Suppression of the Kink Instability for Magnetic Flux Ropes in the Chromosphere. Sol. Phys. 22, 425-433 (1972). DOI 10.1007/BF00148707

76. Savcheva, A.S., van Ballegooijen, A.A., DeLuca, E.E.: Field Topology Analysis of a Longlasting Coronal Sigmoid. ApJ 744, 78 (2012). DOI 10.1088/0004-637X/744/1/78

77. Schrijver, C.J.: Driving major solar flares and eruptions: A review. Advances in Space Research 43, 739-755 (2009). DOI 10.1016/j.asr.2008.11.004

78. Sturrock, P.A.: Maximum energy of semi-infinite magnetic field configurations. ApJ 380, 655-659 (1991). DOI 10.1086/170620

79. Titov, V.S.: Generalized Squashing Factors for Covariant Description of Magnetic Connectivity in the Solar Corona. ApJ 660, 863-873 (2007). DOI 10.1086/512671

80. Titov, V.S., Démoulin, P.: Basic topology of twisted magnetic configurations in solar flares. Astron. Astrophys. 351, 707-720 (1999)

81. Titov, V.S., Hornig, G., Démoulin, P.: Theory of magnetic connectivity in the solar corona. Journal of Geophysical Research (Space Physics) 107, 1164 (2002). DOI 10.1029/ 2001JA000278

82. Török, T., Kliem, B.: Confined and Ejective Eruptions of Kink-unstable Flux Ropes. ApJL 630, L97-L100 (2005). DOI 10.1086/462412

83. Török, T., Kliem, B.: Numerical simulations of fast and slow coronal mass ejections. Astronomische Nachrichten 328, 743 (2007). DOI 10.1002/asna.200710795

84. Török, T., Kliem, B., Titov, V.S.: Ideal kink instability of a magnetic loop equilibrium. Astron. Astrophys. 413, L27-L30 (2004). DOI 10.1051/0004-6361:20031691

85. van der Holst, B., Manchester IV, W., Sokolov, I.V., Tóth, G., Gombosi, T.I., DeZeeuw, D., Cohen, O.: Breakout Coronal Mass Ejection or Streamer Blowout: The Bugle Effect. ApJ 693, 1178 (2009). DOI 10.1088/0004-637X/693/2/1178

86. Webb, D.F., Hundhausen, A.J.: Activity associated with the solar origin of coronal mass ejections. Sol. Phys. 108, 383-401 (1987). DOI 10.1007/BF00214170

87. Woltjer, L.: A Theorem on Force-Free Magnetic Fields. Proceedings of the National Academy of Science 44, 489-491 (1958). DOI 10.1073/pnas.44.6.489

88. Yeates, A.R., Mackay, D.H., van Ballegooijen, A.A.: Modelling the Global Solar Corona II: Coronal Evolution and Filament Chirality Comparison. Sol. Phys. 247, 103-121 (2008). DOI 10.1007/s11207-007-9097-0 
89. Zhang, M., Low, B.C.: Magnetic Energy Storage in the Two Hydromagnetic Types of Solar Prominences. ApJ 600, 1043-1051 (2004). DOI 10.1086/379891

90. Zhang, M., Low, B.C.: The Hydromagnetic Nature of Solar Coronal Mass Ejections. ARAA 43, 103-137 (2005). DOI 10.1146/annurev.astro.43.072103.150602

91. Zhang, Y., Liu, J., Zhang, H.: Relationship between Rotating Sunspots and Flares. Sol. Phys. 247, 39-52 (2008). DOI 10.1007/s11207-007-9089-0 